\title{
Reduced Attitude Control of a Robotic Underwater Vehicle
}

\author{
Lukáš Bláha \\ Department of Cybernetics, University of West Bohemia, Univerzitni 8, 30614, Pilsen, Czech Republic
}

\begin{abstract}
This paper deals with stabilization and reduced attitude control of a robotic underwater vehicle. The vehicle is assumed to be able to perform a full stable rotations around all axes in underwater space, that is why the standard bottom-heavy structure is not used. The system preferably uses a vectored-thrust arrangement and is built as an overactuated system, which enables to gain a better robustness and guarantees a stable controlled motion even if some thruster suddenly stop working. Because the heading angle cannot be measured, the reduced attitude control strategy is designed and the stability of reduced state of the system is proved using perturbation method.
\end{abstract}

\section{Introduction}

In the last few decades, the research of small underwater vehicles has been in the focus of the robotic and offshore engineering community. Especially in recent years the underwater unmanned vehicles have received a new attention that comes from advanced technologies and growing industry [1]. The vehicles for visual and ultrasonic inspection are specific part of that research [2], which very differ from traditional remotely operated vehicle (ROV) [3]. Compared to traditional submarines, they are differently shaped and equipped [4]. These properties have direct connection to accurate motion control. Due to inspection purposes, they has to be able to perform a full attitude maneuvers and therefore the standard auto-stable bottom-heavy structure [5] is not used. On the contrary, full attitude control is suitable for this kind of inspection systems. Research of rigid-body attitude control is therefore motivated not only from space or flight applications.

An accurate dynamic model is a crucial part of designing a stable, controllable and autonomous robotic system. However, the modeling of submerged system dynamics is full of nonlinearities and uncertainties [6]. Moreover the hydrodynamic effects are often coupled
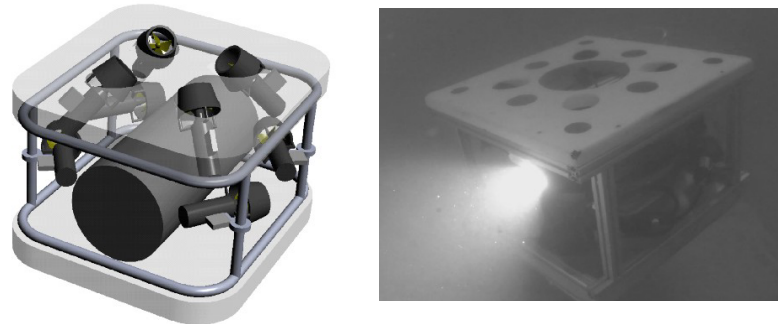

Figure 1: Schematic model of an underwater vehicle with vectored-thrust arrangement and corresponding real prototype. between individual degrees of freedom, and therefore it seems very natural to design a submerged robotic vehicle as symmetric as possible and use an over-actuated control structure, see figure 1. This ensures that the model parameters appear mainly as a diagonal terms of related matrices and the system can be more robust to parametric uncertainties.

This paper deals with modeling and attitude control of a robotic underwater vehicle. The key part of modeling is to get a qualitatively good model, respecting all the major aspects of kinematics and dynamics, important to design of a stable attitude control. The identification techniques of model parameters are detailed described in [7]. Attitude control is based on nonlinear controller with PID structure. It is shown using the perturbation method [8] that for some unknown measurement, the stability of reduced system is ensured.

The paper is organized as follows. Section 2 provides some notations and tools that are used to derive rigid body rotations. Then the vehicle dynamic model is derived and some useful simplifications are described. The benefits of over-actuated vehicle are presented. The parametric model is then used for design of a stable motion controller for a real prototype. Section 4 and 5 deals with attitude control and reduced attitude control of given system with emphasis on the stability and robustness in the entire or reduced working range. The stability for given controller is proved using perturbation method and Lyapunov indirect method. Last section contains some concluding remarks and acknowledgments.

\section{Rigid body kinematics}

This section defines some notation and gives some mathematical background used in the following sections. 
Note that the time dependence is left out for compactness through all paper.

\section{$2.1 \mathrm{SO}(3)$, matrix of rotation, quaternions}

The attitude of rigid body can be represented by rotation matrix $R \in S O(3)$ representing a rotation from body fixed $\mathrm{C}$-frame to inertial $\mathrm{O}$-frame and fulfilling the properties of special orthogonal group

$$
S O(3)=\left\{R \in \mathbb{R}^{3 \times 3}: R^{T} R=I, \operatorname{det} R=1\right\} .
$$

Euler angles, together with any standard set of intrinsic rotations, can be used for specification of rotation matrix $R(\boldsymbol{\theta})$, where the vector of Euler angles is defined as

$$
\boldsymbol{\theta}=[\phi, \theta, \psi]^{T} \text {. }
$$

This specific sequence of 3-2-1 intrinsic rotation is chosen according to limits on measurement of Euler angles, which are then used for a reduced attitude controller, as detailed in Section 4. Consequently the rotation matrix is as follows

$$
R(\boldsymbol{\theta})=\left[\begin{array}{ccc}
c \theta c \psi & s \phi s \theta c \psi-c \phi s \psi & c \phi s \theta s \psi+s \phi s \psi \\
c \theta s \psi & s \phi s \theta s \psi+c \phi c \psi & c \phi s \theta s \psi-s \phi c \psi \\
-s \theta & s \phi c \theta & c \phi c \theta
\end{array}\right]
$$

where $s \cdot=\sin (\cdot), c \cdot=\cos (\cdot), t \cdot=\tan (\cdot)$.

The body angular velocity vector $\boldsymbol{\omega}=\left[\omega_{1}, \omega_{2}, \omega_{3}\right]^{T}$ and Euler rate vector $[\dot{\phi}, \dot{\theta}, \dot{\psi}]^{T}$ are related through a transformation $\dot{\boldsymbol{\theta}}=S(\boldsymbol{\theta}) \boldsymbol{\omega}$, expanding as

$$
\left[\begin{array}{c}
\dot{\phi} \\
\dot{\theta} \\
\dot{\psi}
\end{array}\right]=\left[\begin{array}{ccc}
1 & s \phi t \theta & c \phi t \theta \\
0 & c \phi & -s \phi \\
0 & s \phi / c \theta & c \phi / c \theta
\end{array}\right]\left[\begin{array}{l}
\omega_{1} \\
\omega_{2} \\
\omega_{3}
\end{array}\right], c \theta \neq 0 .
$$

The transformation is undefined for a pitch angle $\theta= \pm \pi / 2$. This is known as a gimbal lock. Note that the matrix $S(\boldsymbol{\theta}) \notin S O(3)$ is not orthogonal.

Other representation of rigid body rotation, such as quaternions, Rodrigues parameters, axis-angle or other parametrization of $R$, are also possible. Their advantages and drawbacks are described for example in [9], [10].

\section{Vehicle dynamics}

The underwater vehicle is a highly nonlinear complex system. The standard general form of mathematical model of submerged rigid body is defined using Kirchhoff equations with external forces representing the thrust of actuators and the hydrodynamic drags

$$
\begin{aligned}
& M \dot{v}-D \dot{\omega}-M v \times \omega+D \omega \times \omega=\sum_{j=1}^{8} T_{j}+R^{T} f_{g}+f_{h} \\
& J \dot{\omega}+D \dot{v}+\omega \times J \omega+\omega \times D v+v \times M v+D \omega \times v= \\
& =r_{g} \times R^{T} m_{b} g e_{z}+\sum_{j=1}^{8} \rho_{j} \times T_{j}+\tau_{h} \\
& \dot{\Gamma}=\Gamma \times \omega
\end{aligned}
$$

where $v, \omega$ indicates the linear and angular velocity of coordinated body $C$-frame, situated in the center of buoyancy $C B, M$ is general mass matrix representing the body and added mass $M=m_{b} I+M_{a d}, \quad J=J_{b}+J_{a d}$ is a tensor of inertia given by body inertia matrix $J_{b}$ and added inertia matrix $J_{a d}$ produced by fluid, $D=m_{b} \hat{r}_{g}$, where ${ }^{\wedge}$ is skew-symmetry operator applied on position vector $r_{g}$ oriented from $C$-frame to center of gravity, $\Gamma=R^{T} e_{z}, f_{g}$ is the force produced by gravity and buoyancy. Hydrodynamic effects $f_{h}, \tau_{h}$ are modeled as an external forces, in general nonlinear form, acting on the vehicle and $T_{j}$ is a vector of thrust force, produced by $\mathrm{j}$-th thruster, mounted on the position $\rho_{j}$, where $j=1,2, \ldots, 8$.

\subsection{Useful simplifications}

The vehicle is built as a neutrally buoyant rigid body. Moreover the center of gravity is coincident ${ }^{1}$ with the center of buoyancy, which results to

$$
\begin{aligned}
& \text { (i) } f_{g}=0, \\
& \text { (ii) } r_{g}=0 \Rightarrow D=0 .
\end{aligned}
$$

From operating purposes it is assumed that the vehicle velocities $v, \omega$ are low enough making the vortex shedding not dominant and viscous effects can be modeled as a drag in form of linear and/or quadratic damping [6]

$$
f_{h}=-\beta_{t r} v, \tau_{h}=-\beta_{r o t} \omega-\gamma_{r o t} \omega|\omega| .
$$

where $\left\{\beta_{t r}, \beta_{r o t}, \gamma_{r o t}\right\} \in \mathbb{R}^{3}$ are positive definite diagonal matrices in form

$$
\begin{aligned}
& \beta_{t r}=\operatorname{diag}\left\{b_{t r l}, b_{t r 2}, b_{t r 3}\right\}, \\
& \beta_{r o t}=\operatorname{diag}\left\{b_{r o t 1}, b_{r o t 2}, b_{r o t 3}\right\}, \\
& \gamma_{r o t}=\operatorname{diag}\left\{c_{r o t 1}, c_{r o t 2}, c_{r o t 3}\right\} .
\end{aligned}
$$

The added mass and added inertia matrices are hard to identify in whole complexity. Considering the vehicle prototype is designed to have almost rounded cubic shape symmetrical about all principal axes, the added matrices can be simplified to the diagonal form

$$
\begin{aligned}
& M=\left(m_{b}+m_{a d}\right) I, \\
& J=J_{b}+\operatorname{diag}\left\{n_{1}, n_{2}, n_{3}\right\}=\operatorname{diag}\left\{J_{1}, J_{2}, J_{3}\right\},
\end{aligned}
$$

and their elements can be identified using experimental identification process [7]. It is reasonable to identify only these diagonal terms of added mass and added inertia matrices, because the stabilizing control law will then be less dependent on generally poorly known parameters.

\subsection{Equation of motion}

Considering only the rotational part of equations (4) and taking the above simplifications into account, the mathematical model of submerged vehicle is in the form

$$
J \dot{\omega}+\omega \times J \omega+\beta_{\text {rot }} \omega+\gamma_{\text {rot }} \omega|\omega|=\sum_{j=1}^{8} \rho_{j} \times T_{j} .
$$

The equations (9) together with kinematics (3) represent the attitude equations of the vehicle.

\footnotetext{
1 It means almost coincident, but the neglecting is reasonable and the inaccuracies are covered by a robust controller.
} 


\subsection{Over-actuated control}

The vehicle prototype is designed to be over-actuated, which means that a number of linearly independent columns of any input matrix $B$ has to be greater than the number of rows of $B$.

Consider input vector $\boldsymbol{\tau}=\left[T_{1}, \ldots, T_{8}\right]^{T}$. The vehicle uses the vectored-thrust arrangement, from which the matrix $B: B \boldsymbol{\tau}=\sum_{j=1}^{8} \rho_{j} \times T_{j}$ is generated as

$$
B=\left[\begin{array}{cccccccc}
\frac{k_{6}}{J_{1}} & \frac{k_{6}}{J_{1}} & \frac{-k_{6}}{J_{1}} & \frac{-k_{6}}{J_{1}} & \frac{k_{8}}{J_{1}} & \frac{k_{8}}{J_{1}} & \frac{-k_{8}}{J_{1}} & \frac{-k_{8}}{J_{1}} \\
\frac{-k_{7}}{J_{2}} & \frac{k_{7}}{J_{2}} & \frac{k_{7}}{J_{2}} & \frac{-k_{7}}{J_{2}} & \frac{-k_{9}}{J_{2}} & \frac{k_{9}}{J_{2}} & \frac{k_{9}}{J_{2}} & \frac{-k_{9}}{J_{2}} \\
\frac{k_{10}}{J_{3}} & \frac{-k_{10}}{J_{3}} & \frac{k_{10}}{J_{3}} & \frac{-k_{10}}{J_{3}} & 0 & 0 & 0 & 0
\end{array}\right] .
$$

The matrix is composed of 5 constant parameters $k_{6}, \ldots, k_{10}$, which define the positions and orientations of mounted thrusters [2].

Taking all possible combinations of arbitrarily chosen $7,6,5$ and 4 thrusters, it can be shown that $B$ still has full rank, and so $\boldsymbol{\tau}$ can affect all degrees of freedom. In case that only arbitrarily 3 thrusters remain operational, the rank of input matrix respects this inequality

$$
\operatorname{rank}(B) \geq 2
$$

and moreover the input matrix $B$ still has full rank with probability equals to 0.785 ,

$$
\begin{aligned}
& P(\operatorname{rank}(B) \equiv 2)=3 / 14, \\
& P(\operatorname{rank}(B) \equiv 3)=11 / 14 .
\end{aligned}
$$

The pseudo-inverse is used for dealing with a number of inputs and a number of states

$$
B B^{+}=I
$$

where $B^{+}$is right inverse of input matrix (10). Using (13) the input $\boldsymbol{\tau}=B^{+} \boldsymbol{u}$ is modified to get the system in form

$$
\begin{aligned}
J \dot{\omega} & =-\omega \times J \omega-\beta_{\text {rot }} \omega-\gamma_{\text {rot }} \omega|\omega|+\boldsymbol{u} \\
\dot{\theta} & =S(\boldsymbol{\theta}) \omega,
\end{aligned}
$$

where $\boldsymbol{u}: \operatorname{dim}(\boldsymbol{u})=3$ is a new input vector.

\section{Attitude control}

\subsection{Attitude measurement}

The measurement of necessary variables is supported by Inertial Measurement Unit (IMU) together with implemented Kalman bias estimating and filtering algorithm. The description of that algorithm is behind the scope of this paper. See [10] or upcoming papers for details.

Let's define an attitude error as

$$
\boldsymbol{e}_{\theta}=\boldsymbol{\theta}-\boldsymbol{\theta}^{*},
$$

where $\boldsymbol{\theta}^{*}(t)=\boldsymbol{\theta}^{*}(0)$ is a constant setpoint. Similarly the angular velocity error is defined as

$$
\boldsymbol{e}_{\omega}=\omega-\omega^{*},
$$

where $\omega^{*}(t)=\omega^{*}(0)$ is a constant setpoint, which will be further specified. Next the attitude error integral is given as

$$
\boldsymbol{e}_{\varphi}=\int_{0}^{t} \boldsymbol{\theta}-\boldsymbol{\theta}^{*} d \tau=\int_{0}^{t} \boldsymbol{e}_{\theta} d \tau
$$

\subsection{Attitude control}

Assume we want to stabilize the vehicle attitude around an equilibrium $\left[\boldsymbol{\theta}^{* T}, \boldsymbol{\omega}^{* T}\right]^{T}, \boldsymbol{\omega}^{*}=\mathbf{0}$. The standard attitude controller uses proportional-derivative feedback, and for large angle maneuvers is in the form

$$
\boldsymbol{u}=-D_{\omega} \boldsymbol{\omega}-S^{T}(\boldsymbol{\theta}) P_{\theta} \boldsymbol{e}_{\theta}, \quad P_{\omega}>0, \quad P_{\theta}>0 .
$$

The stability of closed loop system can be proved by Lyapunov direct method.

Proof of Stability: Consider a Lyapunov function candidate as

$$
V=\frac{1}{2} \omega^{T} J \omega+h\left(\boldsymbol{e}_{\theta}\right),
$$

where $h\left(\boldsymbol{e}_{\boldsymbol{\theta}}\right) \geq 0$ is any positive definite function depending on the attitude error. By the differentiation and using of (14), the change of $V$ can be expressed as

$$
\begin{aligned}
\dot{V} & =\boldsymbol{\omega}^{T} J \dot{\boldsymbol{\omega}}+\dot{\boldsymbol{e}}_{\theta}^{T} \frac{\partial h}{\partial \boldsymbol{e}_{\theta}} \\
& =\boldsymbol{\omega}^{T}\left[-\beta_{r o t} \boldsymbol{\omega}-\gamma_{r o t} \boldsymbol{\omega}|\boldsymbol{\omega}|+\boldsymbol{u}+S^{T}(\boldsymbol{\theta}) \frac{\partial h}{\partial \boldsymbol{e}_{\theta}}\right] \leq 0 .
\end{aligned}
$$

Provided $h\left(\boldsymbol{e}_{\theta}\right)=\frac{1}{2} \boldsymbol{e}_{\theta}{ }^{T} P_{\theta} \boldsymbol{e}_{\theta}, P_{\theta}>0$, then one control law satisfying Lyapunov function candidate and stability condition can be specified as in the (18) which results to

$$
\dot{V}=\omega^{T}\left[-\beta_{r o t} \omega-\gamma_{r o t} \omega|\omega|-D_{\omega} \omega\right] \leq 0 .
$$

The control law (18) is a standard type of attitude rigid body controller, used for example in [11]. Modification for $\mathrm{SO}(3)$ and quaternion domain is presented in [9],[12], [13] and references therein.

Nevertheless the given controller is not suitable for underwater vehicle, where the imperfections in shape, inertia tensors and balancing can very affect the dynamic behavior of system, even the stability.

For improving the robustness of the closed-loop system, and for suppression of attitude errors arising from imperfect balancing of device, it is appropriate to modify the feedback to the form of nonlinear PID type. Then, for example, acting of static gravitational force, arising from imperfect balancing, can be suppressed with desired speed of convergence.

Without loss of generality, assume the nonzero position vector $\boldsymbol{r}_{g}$ is aligned with $\boldsymbol{e}_{3}$ axis of $C$-frame. Then the static force, produced by nonzero distance of $C G$ and $C B$, is in the form

$$
\boldsymbol{r}: \boldsymbol{r}=\boldsymbol{r}_{g} \times R^{T} m_{b} g \boldsymbol{e}_{z}=\left[\begin{array}{c}
-m_{b} g r_{g 3} \cos \theta \sin \phi \\
-m_{b} g r_{g 3} \sin \theta \\
0
\end{array}\right]
$$


The next section describes the proposed type of controller, including the properties of control structure and the proof of stability of the reduced closed loop feedback.

\section{Reduced Attitude control}

The operating conditions of vehicle prototype does not allow to reliably use any heading sensor. Heading is therefore manually commanded by operator, but the reduced attitude stability and full command control has to be fulfilled. Hence is necessary to prove the stability of the reduced controlled system.

\subsection{Reduced attitude kinematics}

As mentioned above the unmeasurable (even unobservable) heading variable means that Euler angle $\psi$ is not known. The kinematic matrix $S(\boldsymbol{\theta})$, used in control loop, depends on parametrization of rotation matrix $R$ and therefore is necessary to find any sequence of intrinsic rotations, which produces the $S(\boldsymbol{\theta})$ independent of $\psi$. One such a parametrization is the sequence of 3-2-1 intrinsic rotation, known as Cardanian angles, which is presented in section 2.1 .

\subsection{Structure and design of reduced controller}

Assuming the balance imperfection in form of (22) and a reduced attitude controller in the form

$$
\boldsymbol{u}=-D_{\omega} \boldsymbol{\omega}-S^{T}(\boldsymbol{\theta}) P_{\theta} \boldsymbol{e}_{\theta}-S^{T}(\boldsymbol{\theta}) I_{\varphi} \boldsymbol{e}_{\varphi},
$$

where $\left\{D_{\omega}, P_{\theta}, I_{\varphi}\right\} \in \mathbb{R}^{3}$ are diagonal matrices in form

$$
\begin{aligned}
& D_{\omega}=\operatorname{diag}\left\{D_{\omega_{1}}, D_{\omega_{2}}, D_{\omega_{3}}\right\}, \\
& P_{\theta}=\operatorname{diag}\left\{P_{\theta_{1}}, P_{\theta_{2}}, 0\right\}, \\
& I_{\varphi}=\operatorname{diag}\left\{I_{\varphi_{1}}, I_{\varphi_{2}}, 0\right\} .
\end{aligned}
$$

The zero diagonal terms reflect the unavailability of heading measurement. Then the two states, used for integral part of controller, are added to equations of motion (14), resulting to expression

$$
\begin{aligned}
& J \dot{\boldsymbol{\omega}}=-\boldsymbol{\omega} \times J \boldsymbol{\omega}-\beta_{r o t} \boldsymbol{\omega}-\gamma_{r o t} \boldsymbol{\omega}|\boldsymbol{\omega}|+\boldsymbol{r}+\boldsymbol{u} \\
& \dot{\boldsymbol{\theta}}=S(\boldsymbol{\theta}) \boldsymbol{\omega} \\
& \dot{e_{\varphi_{1}}}=\phi-\Phi \\
& \dot{e}_{\varphi_{2}}=\theta-\Theta,
\end{aligned}
$$

where the $\Phi, \Theta$ are the given fixed roll and pitch angles. The perturbation analysis [8] is used to prove the stability of reduced attitude controlled system.

Proof of Stability: Because the angle $\psi$ cannot be controlled, let's assume the vehicle spins around the $\boldsymbol{e}_{z}$ axis with a constant angular velocity $\dot{\psi}=\Omega_{\psi}$ and is oriented to given fixed roll and pitch angles $\Phi, \Theta$. The corresponding constant angular velocities $\boldsymbol{\Omega}=\left[\Omega_{1}, \Omega_{2}, \Omega_{3}\right]^{T}$ are given from (3) as

$$
\left[\begin{array}{l}
\Omega_{1} \\
\Omega_{2} \\
\Omega_{3}
\end{array}\right]=\left[\begin{array}{c}
-\Omega_{\psi} \sin \Theta \\
-\Omega_{\psi} \cos \Theta \sin \Phi \\
\Omega_{\psi} \cos \Theta \cos \Phi
\end{array}\right]
$$

Consider a perturbation of system state $\boldsymbol{\omega}=\boldsymbol{\Omega}+\boldsymbol{\delta}_{\omega}$,

$$
\begin{aligned}
& \boldsymbol{\theta}=\left[\Phi+\delta_{\phi}, \Theta+\delta_{\theta}, \Omega_{\psi} t+\psi_{0}\right]^{T}, \\
& \boldsymbol{e}_{\varphi}=\left[\int_{0}^{t} \delta_{\phi} d \tau, \int_{0}^{t} \delta_{\theta} d \tau\right]^{T} .
\end{aligned}
$$

The differentiation implies $\dot{\boldsymbol{\omega}}=\dot{\boldsymbol{\delta}}_{\omega}, \quad \dot{\boldsymbol{\theta}}=\left[\dot{\boldsymbol{\delta}}_{\phi}, \dot{\boldsymbol{\delta}_{\theta}}, \Omega_{\psi}\right]^{T}$, $\dot{\boldsymbol{e}_{\varphi}}=\left[\delta_{\phi}, \delta_{\theta}\right]^{T}$ and the equations of perturbed system arise from substitution to (25), together with (22), (23),

$$
\begin{aligned}
& J \dot{\boldsymbol{\delta}}_{\omega}=-\boldsymbol{\omega} \times J \boldsymbol{\omega}-\beta_{r o t} \boldsymbol{\omega}-\gamma_{r o t} \boldsymbol{\omega}|\boldsymbol{\omega}|+\boldsymbol{r}+\boldsymbol{u} \\
& {\left[\begin{array}{lll}
\dot{\delta_{\phi}} & \dot{\delta}_{\theta} & -
\end{array}\right]^{T}=S\left(\Phi+\delta_{\phi}, \Theta+\delta_{\theta}\right) \boldsymbol{\delta}_{\omega}} \\
& \dot{\boldsymbol{e}_{\varphi}}=\left[\delta_{\phi}, \delta_{\theta}\right]^{T}
\end{aligned}
$$

By omitting the heading state and by linearization around the constant trajectory

$$
x_{\text {traj }}=\left[\Omega_{1}, \Omega_{2}, \Omega_{3}, \Phi, \Theta, \Phi, \Theta\right]^{T},
$$

the dynamics of reduced closed-loop perturbed system can be expressed as

$$
\left[\begin{array}{l}
\dot{\omega}_{1} \\
\dot{\omega}_{2} \\
\dot{\omega}_{3} \\
\dot{\phi} \\
\dot{\theta} \\
\dot{e}_{\varphi_{1}} \\
\dot{e}_{\varphi_{2}}
\end{array}\right]=\boldsymbol{x}_{\text {traj }}+A\left(\Phi, \Theta, \Omega_{\psi}\right)\left[\begin{array}{l}
\delta_{\omega_{1}} \\
\delta_{\omega_{2}} \\
\delta_{\omega_{3}} \\
\delta_{\phi} \\
\delta_{\theta} \\
\delta_{\phi} \\
\delta_{\theta}
\end{array}\right],
$$

where the constant matrix $A$ is parametrized among other model parameters by $\left\{\Phi, \Theta, \Omega_{\psi}\right\}$.

The stability of perturbed system along the trajectory can be checked by Lyapunov indirect method. It is possible to find appropriate controller parameters, guaranteeing that all eigenvalues of $A$ have negative real part and therefore the given controller stabilizes the reduced system, except the neighborhood of gimbal-lock. Figure 2 and 3 show the dependence of maximal real part of eigenvalues for the attitude range $\Phi \in\langle-\pi, \pi\rangle$, $\Theta \in(-\pi / 2, \pi / 2)$, with given spin $\Omega_{\psi}$ and with respect to model parameters and specific $\left\{D_{\omega}, P_{\theta}, I_{\varphi}\right\}$ setting. Note that the limit stable spin $\Omega_{\psi}$ is about $4 \mathrm{rad} / \mathrm{s}$.

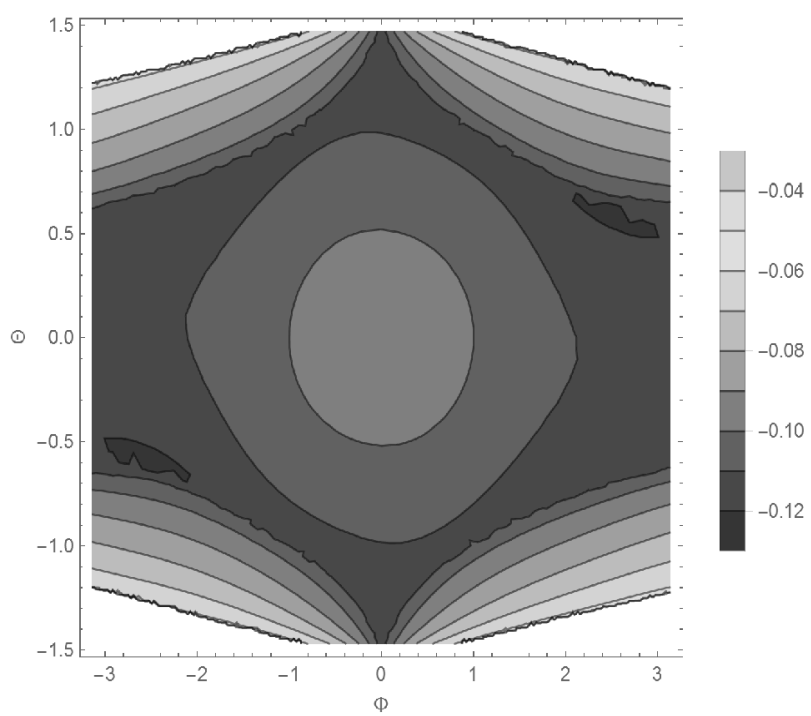

Figure 2: Contour plot of $\max \left(\operatorname{Re}\left[\lambda_{1}, \lambda_{2}, . . \lambda_{7}\right]\right)$ for given pitch and roll parameters $\Phi, \Theta$ and $\Omega_{\psi}=0$. 


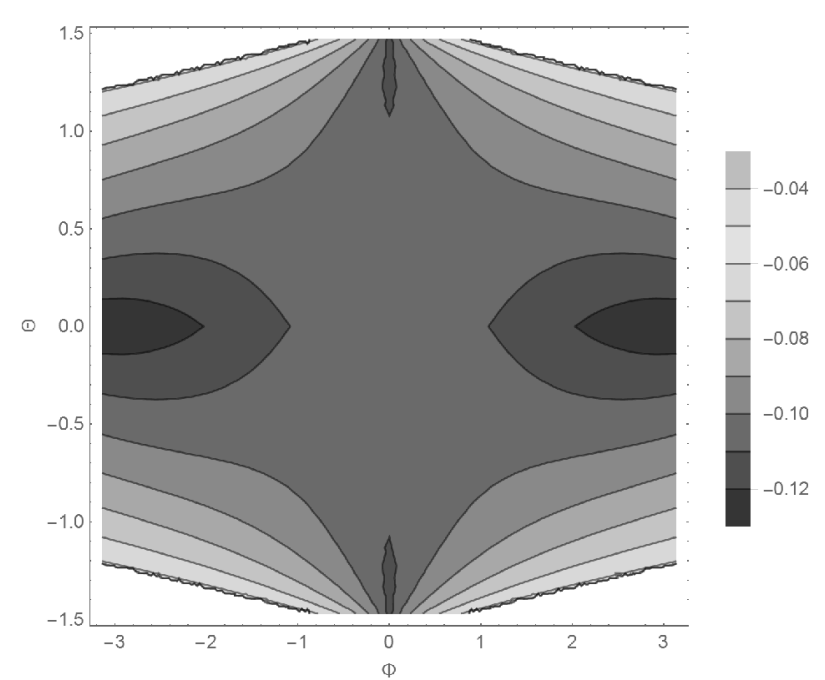

Figure 3: Contour plot of $\max \left(\operatorname{Re}\left[\lambda_{1}, \lambda_{2}, \ldots \lambda_{7}\right]\right)$ for given pitch and roll parameters $\Phi, \Theta$ and $\Omega_{\psi}=1$.

\section{Experimental results}

The controllers were designed to fulfill the stability property of closed loop system as derived above. The parameters of each controller were tuned to get the monotonic response with maximal suitable gain and phase margin on whole attitude range. Figure 4 shows a sample record of attitude control of the real prototype.

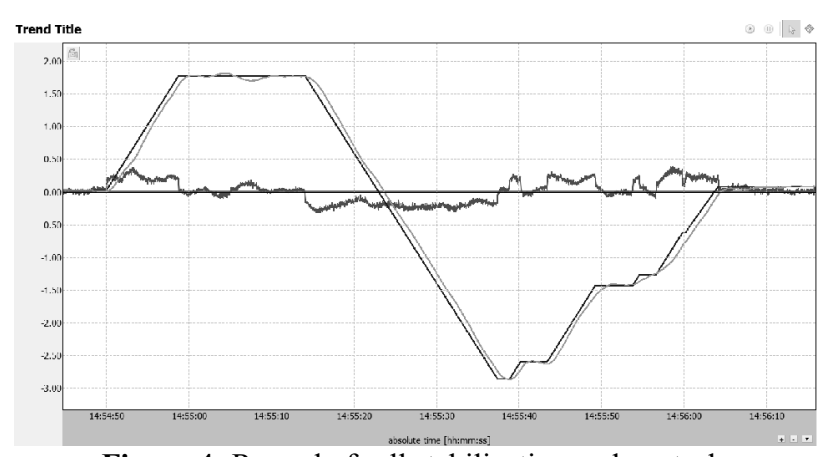

Figure 4: Record of roll stabilization and control.

\section{Conclusions}

In this paper, the mathematical modeling and attitude control of underwater vehicle is presented. On contrary to traditional submarines the robotic inspection vehicles are very differently shaped and equipped, which very affect the required complex motion and stability. Nevertheless, even for full attitude control, some useful simplifications are reasonable, together with over-actuation of system. Their benefits are analyzed. Real applications showed that the model is rich enough to be relevant yet simple to be useful for control design. Attitude control is based on nonlinear controller with PID structure. It is shown that for some unknown measurement, the stability of reduced system is ensured, provided a proper choice of parametrization of rotational matrix and structure of controller. The stability of reduced system was proved using perturbations and Lyapunov indirect method, with domain of attraction in entire reduced working range, except the gimbal-lock. Avoiding this singularity using reduced controller, developed directly on $\mathrm{SO}(3)$, or using quaternions, is a subject of the current research.

\section{Acknowledgment}

This work was supported by the university research program Postdoc, by the project LO1506 of the Czech Ministry of Education, Youth and Sports, and the Technology Agency of the Czech Republic under Grant TE02000103.

\section{References}

1. A.V. Inzartsev, Underwater Vehicles. In-Tech, Austria. (2009).

2. L. Bláha, M. Schlegel and J. Königsmarková, Design and control of underwater vehicle for NDT inspections, 2014 IEEE/OES Autonomous Underwater Vehicles (AUV), Oxford, MS, (2014).

3. T.I. Fossen, Guidance and control of ocean vehicles. Vol. 199. No. 4. New York: Wiley, (1994).

4. S. Mohan, J. Kim, Coordinated motion control in task space of an autonomous underwater vehiclemanipulator system, Ocean Engineering, 104, (2015).

5. E. N. Leonard, Stability of a bottom-heavy underwater vehicle, Automatica 33.3 (1997).

6. W. Wang, Autonomous control of a differential thrust micro rov. (2007).

7. M. Langmajer, L. Bláha, Structural Parameter Identification of a Small Robotic Underwater Vehicle, Mechatronics and Robotics Engineering for Advanced and Intelligent Manufacturing, Springer, (2016).

8. H. K. Khalil, Nonlinear systems, Prentice-Hall, (1996).

9. N. A. Chaturvedi, A. K. Sanyal and N. H. McClamroch, Rigid-Body Attitude Control, in IEEE Control Systems, 31, no. 3, (2011).

10. F. L. Markley, J. L. Crassidis, Fundamentals of Spacecraft Attitude Determination and Control, Springer, (2015).

11. T.I.Fossen, Mathematical models for control of aircraft and satellites, (2011).

12. Y. Yu, S. Yang, M. Wang, C. Li and Z. Li, High performance full attitude control of a quadrotor on $\mathrm{SO}(3)$, IEEE International Conference on Robotics and Automation (ICRA), (2015).

13. T. Lee, M. Leoky and N. H. McClamroch, Geometric tracking control of a quadrotor UAV on $\mathrm{SE}(3), 49$ th IEEE Conference on Decision and Control, (2010). 